Ukai, T., Obayashi, S., Zare-B ehtash, H., Erinc, E., Lo, K.H., and Kontis, K. (2014) Effectiveness of jet location on mixing characteristics inside a cavity in supersonic flow. Experimental Thermal and Fluid Science, 52 . pp. 59-67. ISSN 0894-1777

Copyright @ 2014 Elsevier

A copy can be downloaded for personal non-commercial research or study, without prior permission or charge

Content must not be changed in any way or reproduced in any format or medium without the formal permission of the copyright holder(s)

When referring to this work, full bibliographic details must be given

http://eprints.gla.ac.uk/85724/

Deposited on: 29 A pril 2014

Enlighten - Research publications by members of the University of Glasgow http://eprints.gla.ac.uk 


\title{
Effectiveness of Jet Location on Mixing Characteristics inside a Cavity in Supersonic Flow
}

\author{
Takahiro Ukai*, Shigeru Obayashi, \\ Institute of Fluid Science, Tohoku University, 2-1-1 Katahira, Aoba-ku, Sendai, Miyagi, 980-8577, \\ Japan \\ Hossein Zare-Behtash, Erinc Erdem, Kin Hing Lo, Konstantinos Kontis, \\ The School of MACE, The University of Manchester, Manchester, M13 9PL, UK \\ "Corresponding author: Takahiro Ukai \\ email: ukai@edge.ifs.tohoku.ac.jp
}

Telephone/Fax: + 81-22-217-5257

\begin{abstract}
:
The incorporation of cavities within supersonic combustion chambers is an effective means of slowing down the flow for fuel injection and consequent stable combustion. Understanding the flow physics associated with such flows, especially with the injection of a gas to replicate fuel injection, are essential for the optimum design of supersonic propulsion mechanisms. An experimental investigation was performed on a rectangular open cavity with upstream injection model in a Mach number of 1.9 using a trisonic indraft wind tunnel. A rectangular open cavity of dimensions $L / D=5$, $100 \mathrm{~mm}$ in length $(L)$ and $20 \mathrm{~mm}$ deep $(D)$, was adopted, and it was embedded into the lower wall of the test section. An air jet with a jet-to-free stream momentum flux ratio of $J=1.2,2.7$ and 5.3 was injected upstream of the cavity. To evaluate the effect on mixing and flow stability the jet position, measured from the front edge of the cavity, was varied between $0.1 L$ to $1 L$. The flow field was visualized using schlieren photography, particle image velocimetry, and oil flow measurements. It was found that the mixing characteristic within the cavity when the jet is positioned $0.1 L$ was enhanced independent on the $J$ value because the turbulence intensity of the flow velocity within the cavity was strongly influenced by the jet interaction which lifted the flow from the floor of the cavity compared to the other jet positions. However, the flow over the cavity was unstable at all jet positions. The separation shock formed at the front edge of the cavity oscillated significantly for the case where the jet was located at $0.1 L$ because the separation shock location coincided with the compression shock behind the jet.
\end{abstract}

\section{Keywords:}

Mixing characteristic, Supersonic injection, Cavity, Scramjet 


\section{Introduction}

Development of an optimum supersonic combustion ramjet (scramjet) engines are vital for the realization of hypersonic vehicles [1]. Understanding the effect of the injection system design on the characteristics of the flame stabilization and fuel-air mixing is a key issue for development of scramjets. This is because the residence time of the supersonic freestream within the combustion chamber of a scramjet is extremely short. To understand flow physics inside the combustion chamber, special measuring techniques are utilized. The context of flow diagnostic techniques developed to map surface properties on scramjet combustors, cavities and backward facing steps with jet injection at supersonic speeds [2]. Planar laser-induced fluorescence (PLIF) measuring technique is often used to visualize flames and measure temperature in burning flow fields [3-6].

Studies have already covered the design of injector systems which lead to improve fuel-air mixing characteristics. A flush-mounted fuel injector model which is a simple geometric injection system has been investigated to understand the flow physics of the jet interaction [7-12]. Since a counter-rotating vortex pair appears behind a jet, fuel-air mixing is induced by jet penetration into supersonic crossflow. Multiple injection to enhance the fuel-air mixing characteristics has shown [13-16]. In case of a dual transverse injections system, Lee [13] has shown that an optimal distance between the jets for mixing characteristics exists and this distance is increased as the momentum flux ratio is increased. Pudsey at el. [14] has shown that the multiport jet arrays is more effective for mixing in the near-field around the injection location, although no-improvement in far-field compared to the single jet was found. Impinging shock waves on jetted flows from a single port have been shown to improve the mixing [17-19]. Mai et al. [17] showed that when a shock wave impinges behind an injection slot, the mixing enhances and residence time of the flame is extended.

A cavity model also enhances the characteristics of air mixing [20], and it has been investigated as a flame holding mechanism for the scramjet engine [21-32]. Additionally, the cavity model possesses the feature of comparatively low profile drag compared to a step injector model [33, 34] or strut injector model $[35,36]$, although this feature depends strongly on the cavity geometry. The cavity model also has the advantage of increased net power for the scramjet. A cavity geometry is defined by its length-to-depth ratio $(=L / D)$, it is typically classified as an "open cavity" $(L / D<7-10)$ and "closed cavity" ( $L / D<10-13)$. In the case of the closed cavity, the drag is higher than the open cavity because a shear-layer separated from a front edge of the cavity flows into the cavity and then extremely impacts the aft wall of the cavity resulting in high drag [37]. On the other hand, this shear-layer occurring in the open cavity does not flow into the cavity and most of the shear-layer attaches to the rear edge of the cavity. Hence, an open cavity is generally favored as a flame 
stabilizer due to its low drag properties.

Although the open cavity enhances the characteristic of flame stabilization, this behavior decreases if the flow over the cavity becomes unstable. An acoustic wave originates from the shear-layer due to the velocity differential between the freestream and shear-layer flows, impacting the aft wall of the cavity. Pressure oscillations occur inside the cavity by the repetitive occurrence of compression waves which propagate from the aft wall of the cavity because the acoustic waves are reflected from the aft wall [38]. These pressure oscillations are responsible for the unstable flow over the cavity. In the case where the aft cavity wall is inclined, based on the open cavity, the flow over the cavity becomes steady although the drag is higher than a rectangular open cavity [39]. This is attributed to the higher pressures acting over a larger fraction of the aft wall.

Since the characteristics of flame stabilization changes depending on cavity configuration, injection position, and flow path, the injector model with the rectangular open cavity which possesses low drag might be able to enhance the flame stabilization by modifying the injection position. Previous research has shown that in the case of a cavity with the fuel injector inside the cavity, the open cavity with the aft ramp has a higher performance as a flame holder [22], although the performance depends on injection position [22, 30]. However, in the case of a cavity with upstream fuel injection, a small $L / D$ has better performance for flame stabilization compared to the cavity with the aft ramp. The pressure oscillations over the rectangular cavity disappear if the fluid entrains into the cavity from a side wall of the cavity after fuel is injected [21].

An injection system which has good characteristics for flame stabilization, fuel-air mixing, and low drag is desired for a scramjet engine. It aims at achieving optimum injection utilizing a rectangular open cavity with an upstream injector design. The objective of this experimental investigation is to study the effect of the air mixing and flow stability by varying the injected jet location. The flow stability is visualized using schlieren photography. The air mixing is measured and visualized using the particle image velocimetry (PIV) and surface oil flow techniques for freestream Mach number of 1.9.

\section{Experimental setup}

The experiments were conducted at a Mach number of 1.9 in the Aero-Physics Laboratory trisonic wind tunnel at The University of Manchester. The schematic diagram of the wind tunnel is illustrated in Fig. 1. The tunnel is an intermittent vacuum type with a rectangular test section area measuring $150 \times 215 \times 480 \mathrm{~mm}$ (width $\times$ height $\times$ length). Optical windows are positioned at the side walls of the test section and the upper wall for visualization purposes. A butterfly valve in front of the vacuum tank quickly opens after the pressure in the vacuum tank is evacuated, air flows into the test section 
due to the pressure difference between the atmosphere and vacuum tank. Air moisture is relieved by a heater and desiccants, then the dried air flows into the test section. The tunnel has a stable run time of approximately 5 seconds for the Mach number of 1.9.

The schematic diagram of the cavity geometry is illustrated in Fig. 2. A rectangular open cavity of $L / D=5(L=100 \mathrm{~mm}$ in length and $D=20 \mathrm{~mm}$ in depth) was adopted and it was embedded into the lower wall of the test section. A converging round jet orifice with an exit diameter of $2.2 \mathrm{~mm}$ was vertically machined upstream of the cavity. The jet holes were located $0.1 L(10 \mathrm{~mm}), 0.5 \mathrm{~L}(50 \mathrm{~mm})$ and $1 L(100 \mathrm{~mm})$ from the front edge of the cavity, respectively. High pressure air supplied through a pressure regulator and flexible tubing was used as the jet gas in the experiments. Test conditions are presented in Table. 1. Different jet locations were used to investigate the mixing characteristic and flow stability within the cavity compared to that of the no-jet (baseline) case. When jetted at one location, the air was not supplied at the other locations. The jet pressure was adjusted to provide a jet-to-free stream momentum flux ratio $J=1.2,2.7$ and 5.3 which is defined in Eqn. 1,

$$
J=\frac{\gamma_{j e t} \rho_{j e t} M_{j e t}^{2}}{\gamma_{0} \rho_{0} M_{0}^{2}}
$$

where $\gamma$ is the specific heat ratio, $\rho$ is the density, $M$ is the Mach number and subscripts $O$ and jet refer to freestream and jet conditions.

Schlieren photography [40-44] was employed to visualize the flow field around the cavity in a standard Z-type optical arrangement. A pair of $203.3 \mathrm{~mm}$ diameter parabolic mirrors with $1016 \mathrm{~mm}$ focal length and a 150W Hamamatsu Xenon continuous light source were used. The light generated from the light source is cut off by a slit and is collimated by a parabolic mirror. The collimated light passes through the test section and then is reflected by the second parabolic mirror. The offset angle between the collimated light beam and light source is set at 10 degrees to prevent coma. A knife edge is located at the focus point of the second mirror to adjust the sensitivity. Moreover, a 3-color filter wheel was used in color schlieren mode as a substitute for the knife edge and the slit replaced with a pinhole. High-speed schlieren images were recorded using a high-speed video camera, model Phantom V7.1 with maximum resolution of $800 \times 600$ pixels, Vision research Corp. A frame rate of $2.0 \mathrm{kfps}$ with exposer time of $2 \mu \mathrm{s}$ was set. The color images were recorded using a Canon SLR camera, model EOS-450D with 12 Mpixels resolution. The camera is set to continuous shooting mode at $3.5 \mathrm{fps}$, while the shutter speed is set at a minimum of $0.25 \mathrm{~ms}$.

Particle Image Velocimetry (PIV) was performed to determine the flow velocity and other properties inside the cavity. The PIV setup has been successfully applied in the past studies [19, 45]. A Litron Nano PIV series, Nd:YAG Laser, model LPU550 which possesses a pulse energy of $200 \mathrm{~mJ}$ 
at a repetition rate of $15 \mathrm{~Hz}$ and wavelength of $532 \mathrm{~nm}$ was used for PIV illumination. A laser sheet was positioned above the test section via a laser guide arm and illuminated the center of the cavity along the flow as a planer sheet, see Fig. 3. The laser beam was pulsed as $\Delta t=0.9 \mu$ s interval between two consecutive images so that sufficient displacement for the tracer particles ( 6 pixels, $1 / 5$ of the initial interrogation window) suitable for the current velocity range of $600 \mathrm{~m} / \mathrm{s}$ could be achieved.

Olive oil particles with diameter of $1 \mu \mathrm{m}$ were produced by using TSI's Oil Droplet Generator Model 9307 with $30 \mathrm{~L} / \mathrm{min}$ of aerosol flow rate. Before each run the tunnel inlet was filled with particles and continuous seeding was provided during the tunnel run time. Suitable seeding particle diameter and density are required to capture the flow-tracing accurately. The flow-tracing capability of particles of diameter $d_{p}$ and density $\rho_{p}$ is usually quantified through the particle relaxation time $\tau_{p}$. The theoretical behavior for small spherical particles may be reduced to the modified Stokes drag law [46]. Given the relatively low value of the Mach number and Reynolds number based on the particle diameter, the modified drag relation that takes into account rarefaction effects yields the expression for the particle relaxation time in Eqns. 2 to 4 , where $K n_{d}$ is the Knudsen number based on the particle diameter, and $R e_{d}$ is Reynolds number of the flow around the slipping particles, and $M v$ is the Mach number both evaluated for the maximum particle slip velocity $\Delta V$ [47].

$$
\begin{gathered}
\tau_{p}=d_{p}^{2} \frac{\rho_{P}}{18 \mu_{f}}\left(1+2.7 K n_{d}\right) \\
K n_{d}=1.26 \sqrt{\gamma} \frac{M_{v}}{R e_{d}} \\
R e_{d}=\frac{\rho_{f} \cdot \Delta V \cdot d_{p}}{\mu_{f}}
\end{gathered}
$$

The particle dynamic effects may be further quantified by the Stokes number, $S k$ written in Eqn. $5, S k$ $\ll 1$ has to be satisfied for accurate the flow-tracing. $\delta$ is the boundary layer near the front edge of the cavity. Table 2 shows the timescale and Stokes numbers, $S k \ll 1$ condition was clearly satisfied.

$$
S k=\frac{\tau_{p}}{\tau_{f}} \quad \text { where } \quad \tau_{f}=10 \frac{\delta}{\Delta V}
$$

A LaVision Imager ProX2M CCD camera with $1600 \times 1200$ pixels resolution was used to record scattered light reflected from the particles. The camera equipped with a band-pass filter of $532 \pm 5$ $\mathrm{nm}$, viewed the laser sheet orthogonally. The recorded images were initially divided into $32 \times 32$ pixels interrogation windows and then processed with a cross correlation algorithm using the DaVis 
7.2 software. Then the interrogation windows are refined to $16 \times 16$ pixels. The time averages measurements consisted of a data set of approximate 230 instantaneous vector fields combined from three repeats. With this arrangement, two adjacent velocity vectors were separated by approximately $0.3 \mathrm{~mm}$.

Flow pattern on the floor of the cavity was visualized using oil flow measurements. The formulation used a fluorescent powder suspended in paraffin, oleic acid, and silica gel as the oil material. The oil was poured both inside and ahead of the cavity before a run, and then the oil which is illuminated by UV LEDs, peak wavelength $395 \mathrm{~nm}$, was recorded using the aforementioned Canon camera positioned above the test section during the tunnel run.

\section{Results and discussion}

\subsection{Schlieren photography}

The sequential high-speed schlieren images of the flow field around the cavity are shown in Fig. 4 for each test case of $J=5.7$. Figure 5 shows flow field schematics of the cavity without the jet (No jet) and with the jet $(0.1 L)$. Since higher penetration into the main cross flow occurred as the jet-to-free stream momentum flux ratio increased, the angle of the bow shock in front of the jet changed for different $J$ values, but large density changes did not appear around the cavity. Although the gap shock occurred from the upper wall of the test section because gap of parts between the supersonic insert and upper wall existed, the gap shock appears to be weak and does not influence the result.

Shock waves appeared above the shear-layer, the flows over the cavity became unstable in all cases even when air was jetted from the injection because an acoustic wave generated from the shear-layer over the cavity attached to the rear edge of the cavity in all cases. However, even though the shear-layer impacted the aft wall in this experiment, the impacting shear-layer can be prevented if a shock wave impinges over a cavity. This is because that the shock wave impinging on the shear-layer lift [23].

Large density changes did not appear in each case although the separation shock from the front edge of the cavity oscillated only for CASE 7. The color schlieren images are shown in Fig. 6. The layout of the color filter used is shown in Fig. 6 (a). The front edge of the cavity is zoomed in to investigate the oscillation of the separation shock from the front edge in CASE 7. A shock wave occurs downstream of the Mach disk which is defined as the compression shock. The oscillation of the separation shock is attributed to the impingement of this compression shock. The compression shock has a different shock angle compared with the separation shock from the front edge (Fig. 6 (c)). 
Although the compression shock did not impinge in CASE 8 immediately since the jet was located away from the front edge, it impinged on the separation shock in CASE 7.

\subsection{PIV}

Figure 7 shows time averaged velocity in the $\mathrm{x}$-direction which is the main flow direction. Averaged streamlines are also presented in the same in Fig. 7. The spatial coordinates are normalized with the cavity depth, $D$. The maximum velocity contour was limited to $500 \mathrm{~m} / \mathrm{s}$ to visualize the flow inside the cavity clearly. Although a strong contour in CASE 0 appeared above the front and rear edge due to a reflection of the laser sheet from the model surface, it does not affect the velocity measurement over and inside the cavity. In case of the open cavity without a jet, a pair of vortex inside the cavity appears at the fore and aft wall [28]. In this experiments also, a pair of vortices appeared inside the cavity in CASE 0 (No jet).

If the flow behind the jet flows into the cavity, the vortex does not attach to the aft wall of the cavity and the strength of this vortex also increases. In CASE 5 and $8(0.5 L)$, the flow over the cavity flows into the cavity easily because the flow, which is slightly faster than the velocity inside the cavity, at an inlet of the cavity was induced by jet interaction. On the other hand, in CASE $2(0.5 L, J$ $=1.2$ ), the large part of flows did not flow into the cavity because of the low jet penetration, and the large vortex appeared inside the cavity near the aft wall.

Jet interaction does not significantly affect the flows inside the cavity if the jet position is further away from the front edge of the cavity. The flow velocity and flow structures inside the cavity in CASE 0 resembled that in CASE 3, 6 and $9(1 L)$ even though the jet penetrations were different in each case. A large vortex appeared towards the aft wall of the cavity in CASE 4 and $7(0.1 L)$. This is because the flow velocity behind the jet is reduced due to the interaction with the jet compared to the main flow velocity, and the flow inside the cavity is lifted up. However, the vortex did not grow at the aft wall of the cavity in CASE $1(0.1 L, J=1.2)$ even when the jet position was close to the front edge of the cavity. Figure 8 shows the height from the floor of the cavity to the slip line of $400 \mathrm{~m} / \mathrm{s}$ over the cavity at $x / D=4$ position. The low flow velocity area increased in all jet positions as the $J$ value increased because the low flow velocity area behind the jet did not widely occur when the low jet penetration. Thus in CASE $1(0.1 L, J=1.2)$, flows inside the cavity were not able to flow out from the cavity (Fig. 7 (b)). In the jet position of 0.5L (CASE 2, 5 and 8), since the flows over the cavity flowed into the cavity, the height became low (Fig. 8), compared to the jet positions of $0.1 \mathrm{~L}$ and $1 L$. In the case of the jet positions away from the front edge of the cavity, the flows might not flow out from the cavity even if the height was large because the main flow which is high in velocity was rounded to the shear-layer over the cavity. 
Figure 9 shows the velocity contour of root-mean-square for the x-direction. In CASE 7, the turbulence intensity around the cavity was strongest and its area widest, this implies the enhancement of the mixing characteristic. The turbulence intensity inside the cavity near the fore wall in CASE 5 and 8 was weaker than the other cases because the strong single vortex which occurs near the fore wall. On the other hand, in CASE $2(0.5 L, J=1.2)$, the turbulence intensity at the shear-layer was strong compared to CASE $5(0.5 L, J=2.7)$ and $8(0.5 L, J=5.7)$. The turbulence intensity inside the cavity and the shear-layer in CASE 3, 6 and $9(1 L)$ resembled that in CASE 0 (No jet). In CASE 1, 4 and $7(0.1 L)$, the turbulence intensity was strong both over and inside the cavity. Hence, the mixing characteristic around the cavity can be enhanced at the jet position of $0.1 \mathrm{~L}$ independent on the $J$ value and the mixing characteristic around the cavity does not enhance at the jet position of $1 L$.

\subsection{Oil flow}

Figure 10 shows the flow pattern on the floor of the cavity. The flow pattern in CASE 0 (No jet) generally resembles the results of Sakamoto et al. [23]. Flow structure schematics around the cavity are shown in Fig. 11.

In CASE 0, two vortices appear on the cavity profile along the model centerline. The flow between these vortices might be lifted up from the floor of the cavity due to the interaction between these vortices. In CASE 8, since the single vortex was strong, the flows in front of the vortex were also separated from the floor. Thick lines on the floor indicate the flow being lifted. The distance of the single vortex from front edge of the cavity in the PIV results correlate well with the distance marked $X$ from the end of streaks from front edge in the oil flow results (Fig. 10 (c)). The distance of the flow lifted area (the thick line) is shown in Fig. 12. Measuring the distance of thick line on the centerline of the cavity, the average values are obtained from the five separate images separated by a time interval of $0.25 \mathrm{~ms}$. The distance increased as the jet position approached to the front edge of the cavity, although the distances at the jet position of $100 \mathrm{~mm}(1 L)$ were smaller than that in the no-jet. In addition, it seems that the large part of the flows lifted from the floor of the cavity easily if the $J$ value increased. The air mixing does not necessarily become enhanced if the distance increases because these values do not mean the turbulence intensity.

It is also important for the fuel-air mixing to evaluate the vertical flow structures within the cavity. The short dotted lines in the Fig. 10 indicate the vortex pattern on the floor of the cavity. The flow path was obtained from the images separated by a time interval of $0.25 \mathrm{~ms}$. A vortex pair occurred near the fore wall of the cavity on the model centerline symmetrically. In CASE 7, air mixing was enhanced near the fore wall of the cavity. The widely mixing area appeared because the vortices 
apart occurred and it appeared near the model centerline compared to the other cases. On the other hand, in CASE 0, 8 and 9, the vortex pair occurred near the side wall of the test section.

\section{Conclusion}

An experimental investigation was performed on a rectangular open cavity with upstream injector model in a freestream Mach number of 1.9. Flow stability and mixing characteristics within and over the cavity when the jet injection position was varied has been studied. Using schlieren photography, PIV and oil flow, the flow was examined. Jet injection positions from the front edge of the cavity of $0.1 L(10 \mathrm{~mm}), 0.5 L(50 \mathrm{~mm})$ and $1 L(100 \mathrm{~mm})$ were used $(L=$ length of the cavity), and an air of a jet-to-free stream momentum flux ratio $J=1.2,2.7$ and 5.3 was jetted.

The flows over the cavity were unstable in all cases with and without air being jetted from the orifice. Although a separation shock from the front edge of the cavity oscillated only when at the jet position of $0.1 \mathrm{~L}$. This was attributed to the separation shock impinging on the compression shock behind the jet. Large density gradients were not observed for each jet position and $J$ values. Enhanced mixing characteristics around the cavity, for the jet injection position of $0.1 L$, was observed independent on the $J$ value. This is because that turbulence intensity for flow velocity around the cavity was strong due to the jet interaction, and the flow was strongly lifted from the floor of the cavity compared to the other jet positions.

The rectangular open cavity of dimensions $L / D=5$ with a single jet positioned $10 \mathrm{~mm}$ from the front edge of the cavity exhibited higher performance in the mixing characteristics at a Mach number of 1.9. However, the characteristics of flame stabilization might be lost because flow becomes unstable over the cavity. If the flow instability can be improved by using shock impingement or an inclined aft cavity wall, the present injection system can improve the effectiveness of scramjet combustion.

\section{Acknowledgment}

The first author was supported from Tohoku University Global COE Program for the cooperative research with the laboratory under Professor K. Kontis. The authors are indebted to the technical and administrative staff of the School of MACE at The Manchester University, especially Mr. Lee Paul for the manufacture of the models. 


\section{References}

1. J. P. Drummond, M. Bouchez, C. R. McClinton, Overview of NATO background on scramjet technology, NASA techdoc 20060020216, 2006.

2. K. Kontis, Y. Syogenji, N. Yoshikawa, Surface thermometry by laser-induced fluorescence of Dy3 +: YAG, Aeronautical Journal 106 (1062) 453-457.

3. R. R. Boyce, N. R. Mudford, J. R. McGuire, OH-PLIF visualisation of radical farming supersonic combustion flows, Shock Waves 22 (2012) 9-21.

4. M. -B. Sun, H. Geng, J. -H. Liang, Z. -G. Wang, Mixing Characteristics in a Supersonic Combustor with Gaseous Fuel Injection Upstream of a Cavity Flameholder, Flow Turbulence and Combustion 82 (2009) 271-286.

5. C. T. Johansen, C. D. McRae, P. M. Danehy, E. Gallo, L. Cantu, G. Magnotti, A. Cutler, R. D. Rockwell, C. P. Goyne, J. C. McDaniel, OH PLIF Visualization of the UVa Supersonic Combustion Experiment: Configuration A, AIAA paper 2012-2887 (2012).

6. D. J. Micka, J. F. Driscoll, Reaction Zone Imaging in a Dual-Mode Scramjet Combustor Using CH-PLIF, AIAA paper 2008-5071 (2008).

7. E. Erdem, K. Kontis, Numerical and experimental investigation of transverse injection flows, Shock Waves 20 (2) (2010) 103-118.

8. E. Erdem, K. Kontis, Non-Plasma and Plasma Transverse Jets in Hypersonic Cross Flow, AIAA paper 2009-7253 (2009).

9. W. M. VanLerberghe, J. G. Santiago, J. C. Dutton, R. P. Lucht, Mixing of a Sonic Transverse Jet Injected into a Supersonic Flow, AIAA Journal 38 (3) (2000) 470-479.

10. S. Kawai, S. K. Lele, "Large-Eddy Simulation of Jet Mixing in Supersonic Crossflows," AIAA Journal 48 (9) (2010) 2063-2083.

11. M. R. Gruber, A. S. Nejad, T. H. Chen, J. C. Dutton, Transverse Injection from Circular and Elliptic Nozzles into a Supersonic Crossflow, Journal of Propulsion and Power 16 (3) (2000) 449-457.

12. S. -H. Won, I. -S. Jeung, B. Parent, J. -Y. Choi, Numerical Investigation of Transverse Hydrogen Jet into Supersonic Crossflow Using Detached-Eddy Simulation, AIAA Journal 48 (6) (2010) $1047-1058$

13. S. - H. Lee, Characteristics of Dual Transverse Injection in Scramjet Combustor, Part 1: Mixing, Journal of Propulsion and Power 22 (5) (2006) 1012-1019.

14. A. S. Pudsey, R. R. Boyce, Numerical Investigation of Transverse Jets Through Multi-Port Injector Arrays in a Supersonic Crossflow, AIAA Paper 2008-2517 (2008). 
15. L. S. Jacobsen, J. A. Schetz, W. F. Ng, Flowfield near a Mulitiport Injector Array in a Supersonic Flow, Journal of Propulsion and Power 16 (2) (2000) 216-226.

16. S. K. Cox-Stouffer, M. R. Gruber, Effects of Injector Yaw on Mixing Characteristics of Aerodynamic Ramp Injectors, AIAA Paper 99-0086 (1999).

17. T. Mai, Y. Sakimitsu, H. Nakamura, Y. Ogami, T. Kudo, H. Kobayashi, "Effect of the incident shock wave interacting with transversal jet flow on the mixing and combustion," Pro. Combust. Ins. 33 (2) (2011) 2335-2342.

18. J. A. Schetz, L. Maddalena, S. K. Burger, Molecular Weight and Shock-Wave Effects on Transverse Injection in Supersonic Flow, Journal of Propulsion and Power 26 (5) (2010) 1102-1113.

19. E. Erdem, S. Saravanan, J. Lin, K. Kontis, Experimental Investigation of Transverse Injection Flowfield at Mach 5 and the Influence of Impinging Shock Wave, AIAA Paper 2012-5800 (2012).

20. A. Zang, T. Tempel, K. Yu, S. G. Buckley, Experimental Characterization of Cavity-Augmented Supersonic Mixing, AIAA paper 2005-1423 (2005).

21. K. Yu, K. J. Wilson, R. A. Smith, K. C. Schadow, Experimental Investigation on Dual-Purpose Cavity in Supersonic Reacting Flows, AIAA paper 98-0723 (1998).

22. M. R. Gruber, J. M. Donbar, C. D. Carter, K.-Y. Hsu, Mixing and Combustion Studies Using Cavity-Based Flameholders in a Supersonic Flow, Journal of Propulsion and Power 20 (5) (2004) 769-778.

23. K. Sakamoto, K. Matsunaga, K. Fujii, Y. Tamura, Experimental Investigation of Supersonic Internal Cavity Flows, AIAA paper 95-2213 (1995).

24. M. Gruber, J. Donbar, T. Jackson, T. Mathur, D. Eklund, F. Billig, Performance of an Aerodynamic Ramp Fuel Injector in a Scramjet Combustor, AIAA paper 200-3708 (2000).

25. T. Mathur, G. Streby, M. Gruber, K. Jackson, J. Donbar, W. Donaldson, T. Jackson, C. Smith, F. Billig, Supersonic Combustion Experiments with a Cavity-Based Fuel Injector, AIAA paper 99-2102 (1999).

26. C. D. Ghodke, J. Pranatharthikaran, G. J. Retaureau, S. Menon, Numerical and Experimental Studies of Flame Stability in a Cavity Stabilized Hydrocarbon-Fueled Scramjet, AIAA paper 2011-2365 (2011).

27. R. A. Baurle, T. Mathur, M. R. Gruber, K. R. Jackson, A Numerical and Experimental Investigation of a Scramjet Combustor for Hypersonic Missile Applications, AIAA paper 1998-3121 (1998).

28. C. W. Allen, P. I. King, M. R. Gruber,C. D. Carter, K. -Y. Hsu, Fuel-Air Injection Effects On 
Combustion In Cavity-Based Flameholders In a Supersonic Flow, AIAA paper 2005-4105 (2005).

29. S. G. Edens, P. I. King, M. R. Gruber, K. -Y. Hsu, Performance Measurements of Direct Air Injection in a Cavity-Based Flameholder for a Supersonic Combustor, AIAA paper 2006-4861 (2006).

30. C. C. Rasmussen, J. F. Driscoll, C. D. Carter, K. -Y. Hsu, Characteristics of Cavity-Stabilized Flames in a Supersonic Flow, Journal of Propulsion and Power 21 (4) (2005) 765-768.

31. K. -C. Lin, C. -J. Tam, K. Jackson, Study on the Operability of Cavity Flameholders inside a Scramjet Combustor, AIAA paper 2009-5028 (2009).

32. C. Lada, K. Kontis, Experimental Studies on Transitional and Closed Cavity Configurations Including Flow Control, Journal of Aircraft 47 (2) (2010) 723-730.

33. T. -H. Kim, T. Obara, M. Yoshikawa, S. Ohyagi, Experimental study on the flow field behind a backward-facing step using a detonation-driven shock tunnel, Shock Waves 15 (2006) 1-12.

34. K. Kontis, Surface Heat Transfer Measurements Inside a Supersonic Combustor by Laser-Induced Fluorescence, Journal of Thermophysics and Heart Transfer 17 (3) (2003) $320-325$

35. X. Y. Chang, L. H. Chen, H. B. Gu, G. Yu, Experimental studies on model scramjet in a Mach 6 high enthalpy free-jet wind tunnel," Proceedings of the 24th International Symposium on Shock Waves Beijing, 2005, pp.891-896.

36. G. Y. Anderson, P. G. Reagon, P. B. Gooderum, W. R. Russin, Experimental Investigation of a Swept-Strut Fuel-Injector Concept for Scramjet Application, NASA TN D-8454 (1977).

37. D. E. Nestler, A. R. Saydah, W. L. Auxer, Heat transfer to steps and cavities in hypersonic turbulent flow, AIAA paper 68-973 (1968).

38. W. Li, T. Nonomura, K. Fujii, Effects of shear-layer characteristic on the Feedback-loop Mechanism in supersonic open cavity flows, AIAA paper 2011-1218 (2011).

39. M. R. Gruber, R. A. Baurle, T. Mathur, K. -Y. Hsu, Fundamental Studies of Cavity-Based Flameholder Concepts for Supersonic Combustors,” Journal of Propulsion and Power 17 (2001) 146-153.

40. K. Kontis, C. Lada, H. Zara-Behtash, Effect of dimples on glancing shock wave turbulent boundary layer interactions, Shock Waves 17 (5) (2008) 323-335.

41. K. Kontis, R. An, H. Zare-Behtash, D Kounadis, Head-on collision of shock wave induced vortices with solid and perforated walls, Physics of Fluids 20 (2008) 1-17.

42. H. Zare-Behtash, N. Gongora-Orozco, K. Kontis, Effect of primary jet geometry on ejector performance: A cold-flow investigation, International Journal of Heat and Fluid Flow 32, (2011) 
596-607.

43. R. Erfani, H. Zare-Behtash, K. Kontis, Influence of shock wave propagation on dielectric barrier discharge plasma actuator performance, J. Phys. D: Appli. Phys. 45, 225201 (2012).

44. J. S. Oh, O. T. Olabanji, C. Hale, R. Mariani, K. Kontis, J. W. Bradley, Imaging gas and plasma interactions in the surface-chemical modification of polymers using micro-plasma jets, J. Phys. D: Appl. Phys. 44 (2011) 1-6.

45. R. Erfani, H. Zare-Behtash, K. Kontis, Plasma actuator: influence of dielectric surface temperature, Experimental Thermal and Fluid Science 42 (2012) 258-264.

46. A. Melling, Tracer Particles and Seeding for Particle Image Velocimetry, Meas. Sci. Technol. 8 (1997) 1406-1416.

47. F. Scarano, Overview of PIV in Supersonic Flows, Topics in Applied Physics 112 (2008) 445-463. 


\section{Figures}

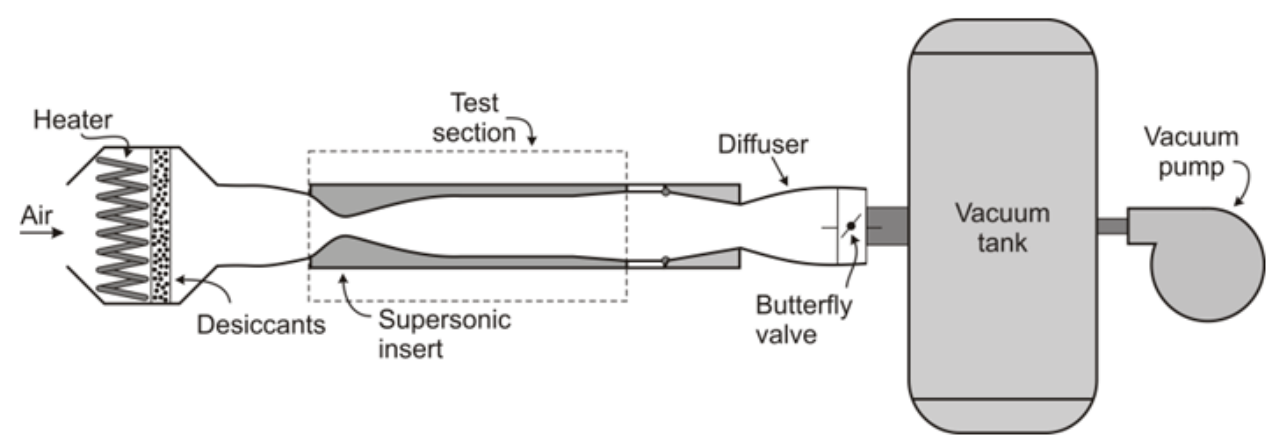

Figure 1: The Aero-Physics Laboratory trisonic wind tunnel schematic layout.

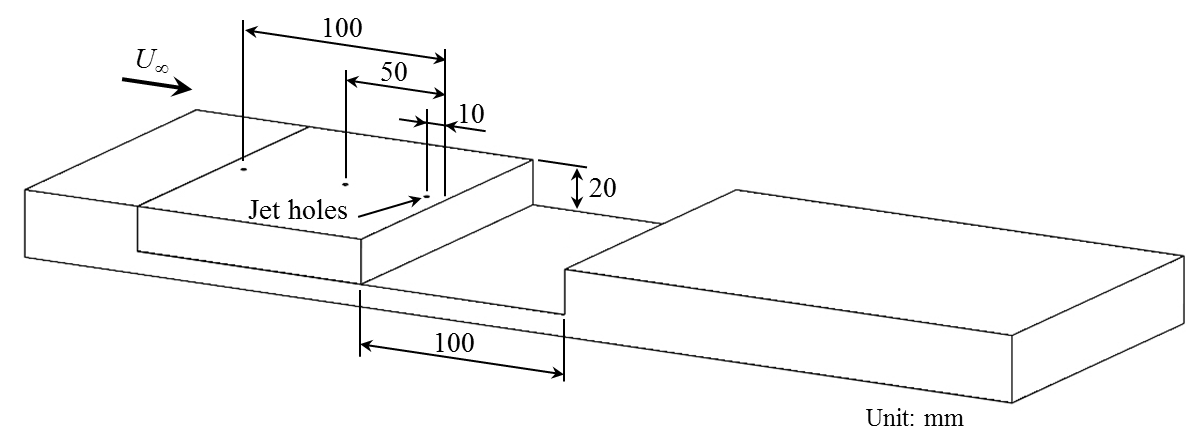

Figure 2: The cavity configuration.

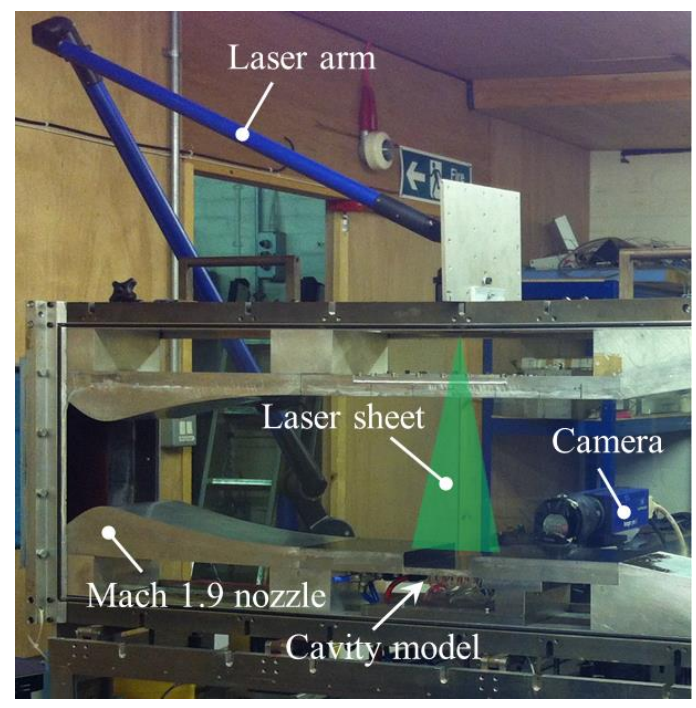

Figure 3: PIV setup on the trisonic wind tunnel. 


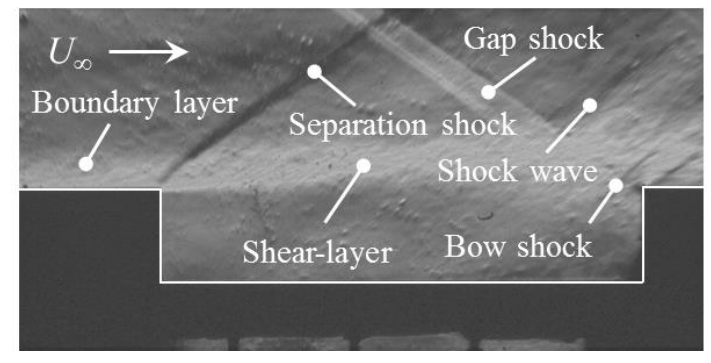

(a) CASE 0 (No jet)

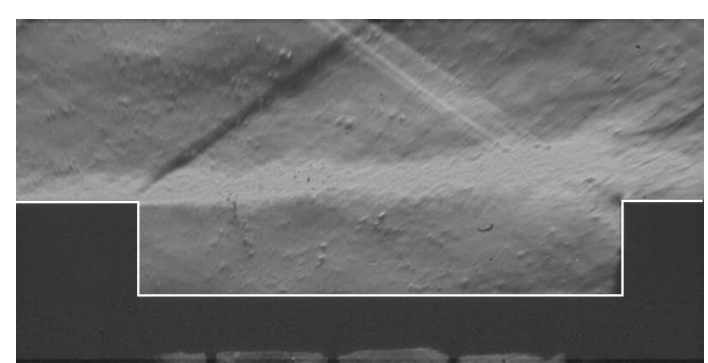

(c) CASE $8(0.5 L, J=5.3)$

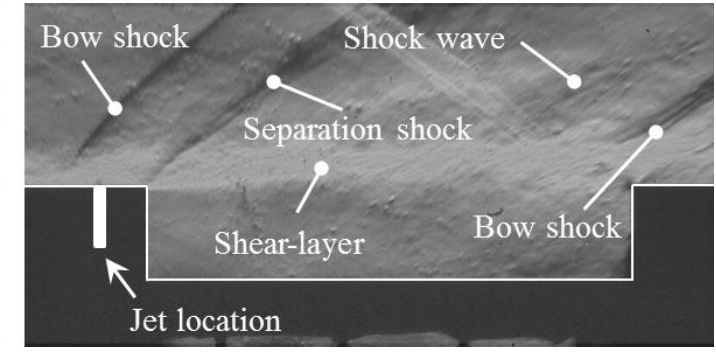

(b) CASE $7(0.1 L, J=5.3)$

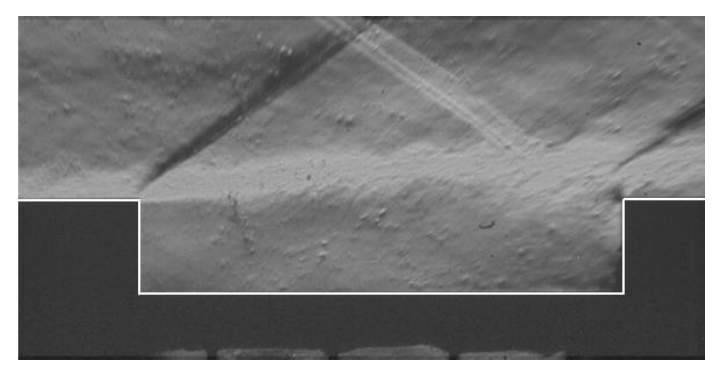

(d) CASE $9(1 L, J=5.3)$

Figure 4: Sequential high-speed schlieren images with 4 test cases.

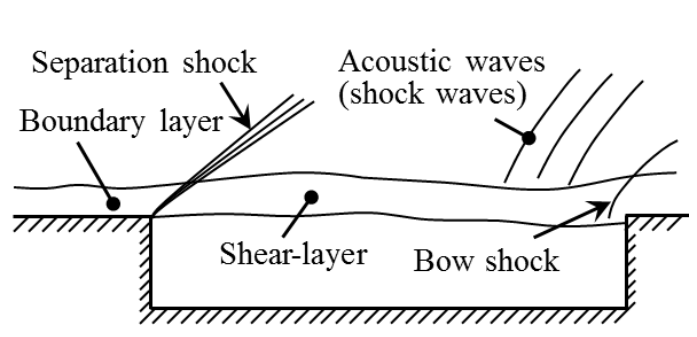

(a) No jet

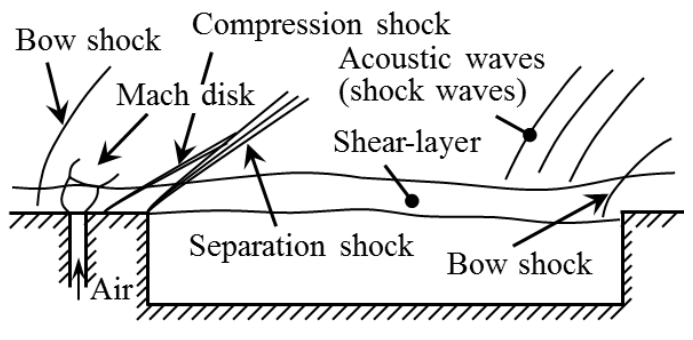

(b) Jet penetration at $10 \mathrm{~mm}$ from the front edge of the cavity $(0.1 L)$

Figure 5: Flow field schematics of the cavity without the jet and with the jet. 


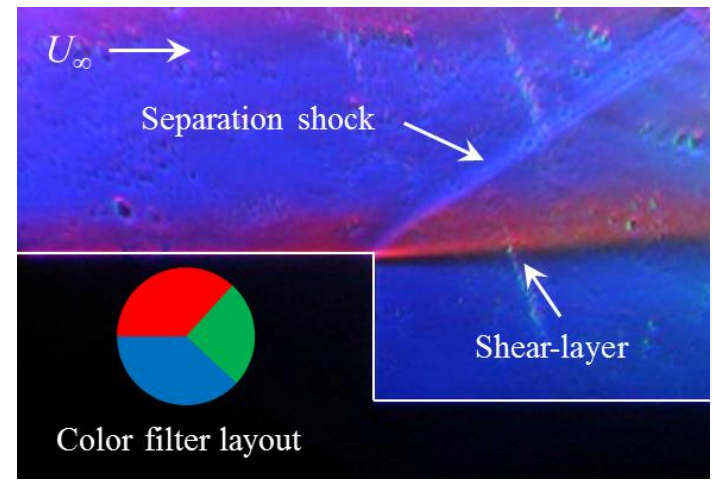

(a) CASE 0 (No jet)

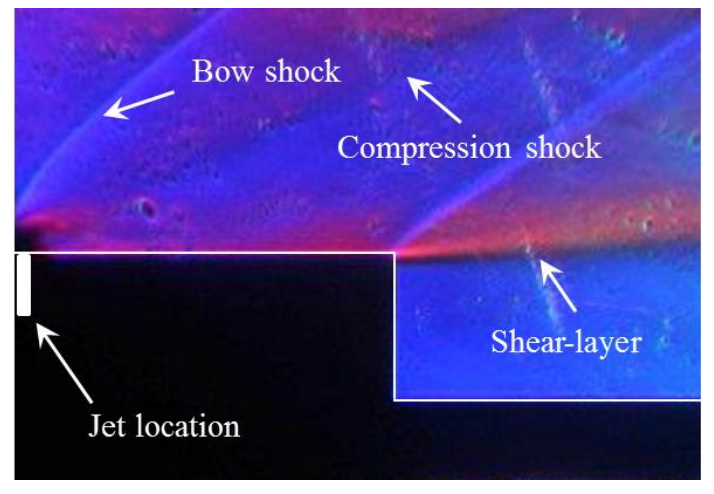

(c) CASE $8(0.5 L, J=5.3)$

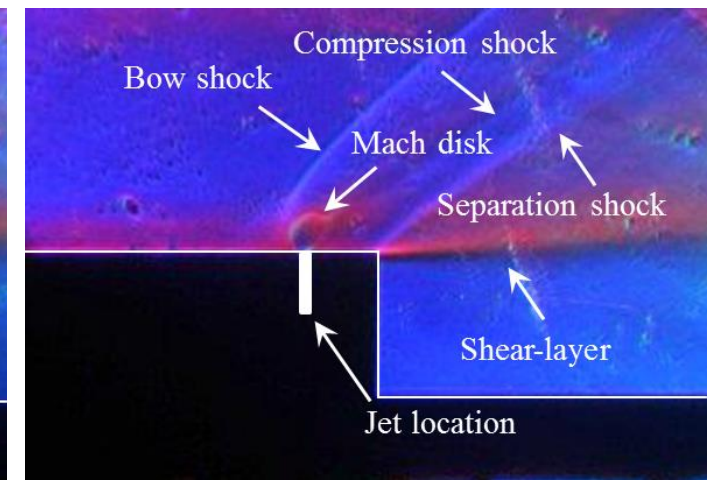

(b) CASE $7(0.1 L, J=5.3)$

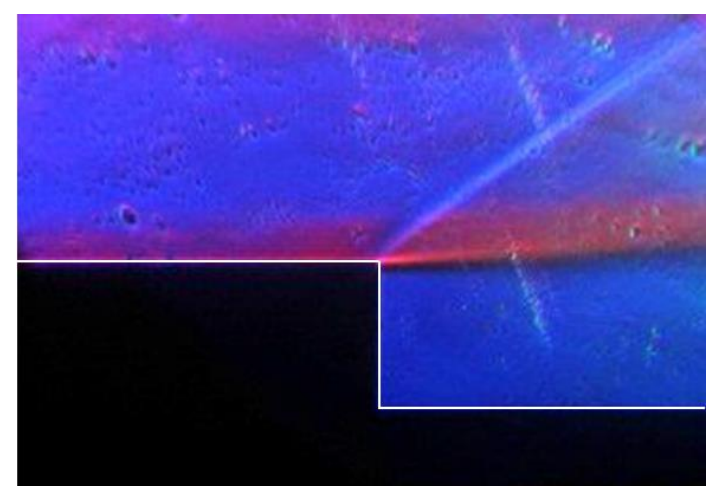

(d) CASE $9(1 L, J=5.3)$

Figure 6: Color schlieren image with 4 test cases. 


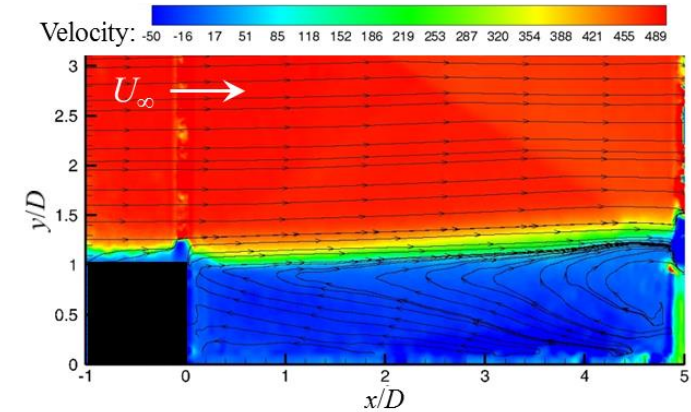

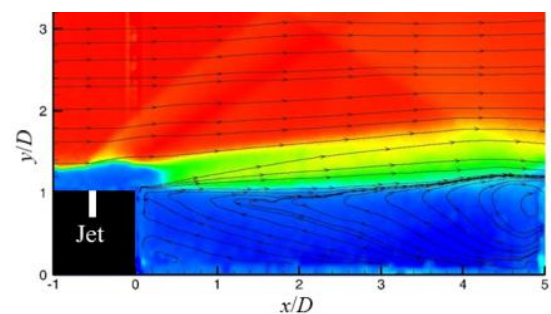

(b) CASE $1(0.1 L, J=1.2)$

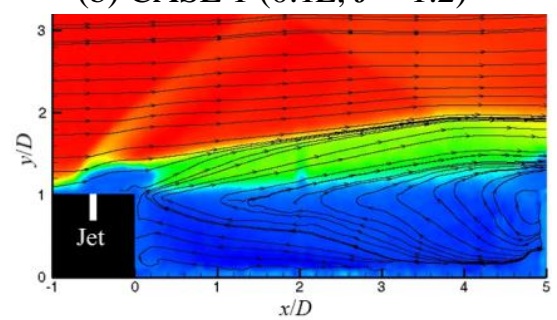

(e) CASE $4(0.1 L, J=2.7)$

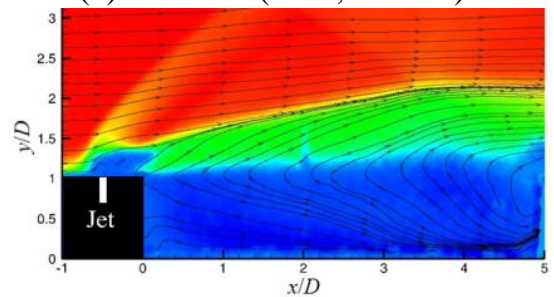

(h) CASE $7(0.1 L, J=5.3)$

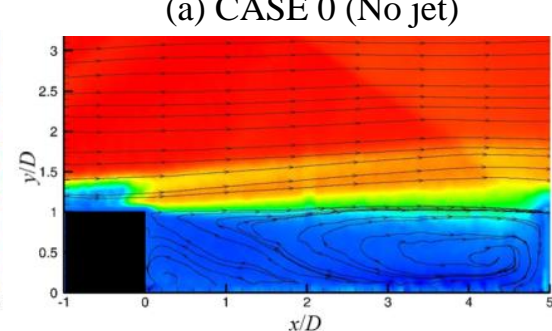

(c) CASE $2(0.5 L, J=1.2)$

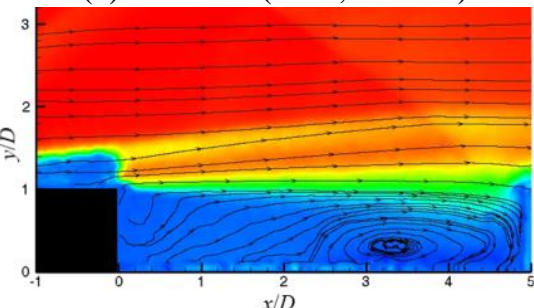

(f) CASE $5(0.5 L, J=2.7)$

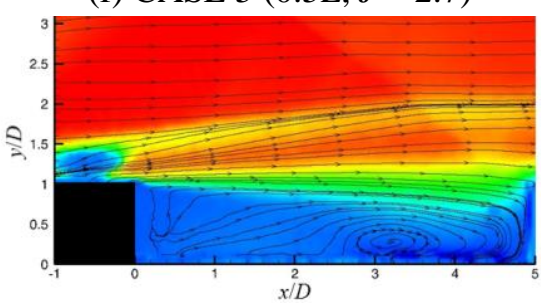

(i) CASE $8(0.5 L, J=5.3)$

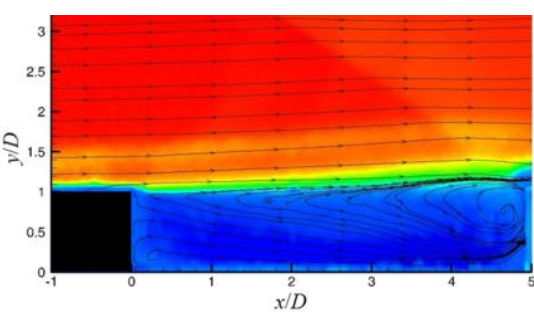

(d) CASE $3(1 L, J=1.2)$

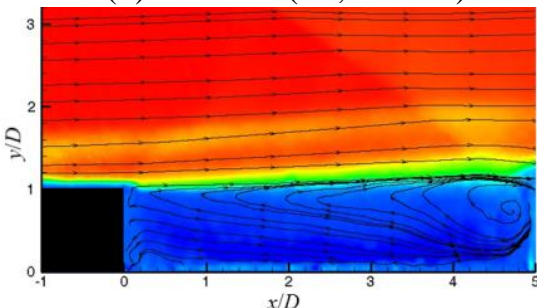

(g) CASE $6(1 L, J=2.7)$

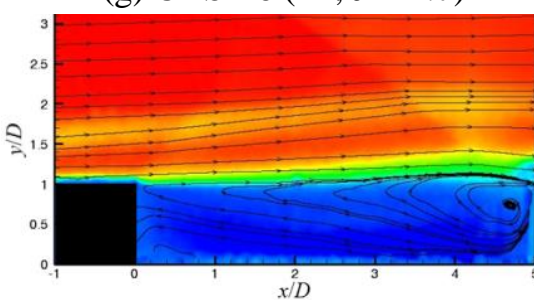

(k) CASE $9(1 L, J=5.3)$

Figure 7: Time averaged flow field velocity contours.

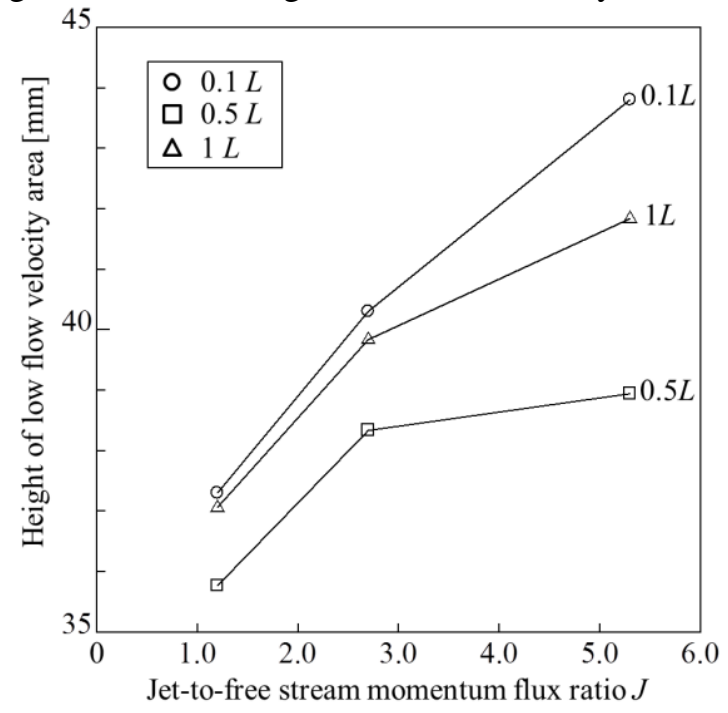

Figure 8: The height from the floor of the cavity to the slip line of $400 \mathrm{~m} / \mathrm{s}$ over the cavity at $x / D=4$ position. 


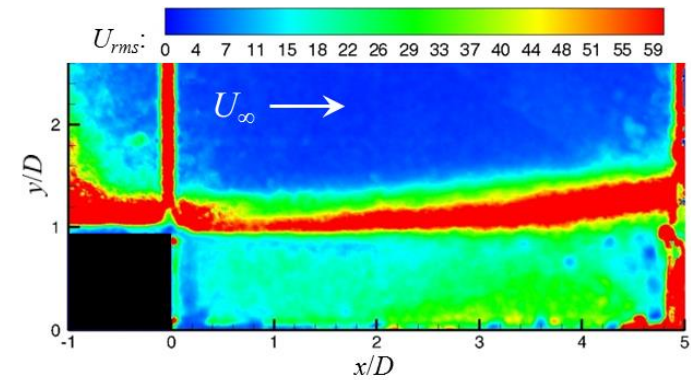

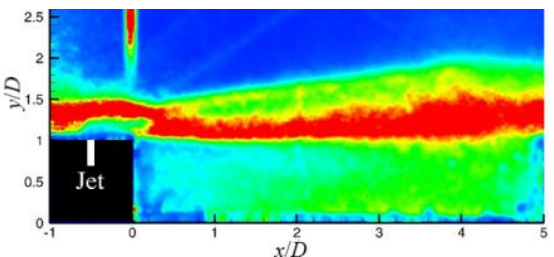

(b) CASE $1(0.1 L, J=1.2)$

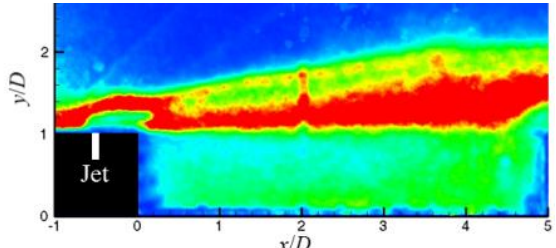

(e) CASE $4(0.1 L, J=2.7)$

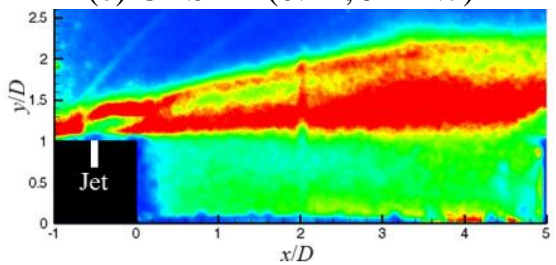

(h) CASE $7(0.1 L, J=5.3)$ (a) CASE 0 (No jet)

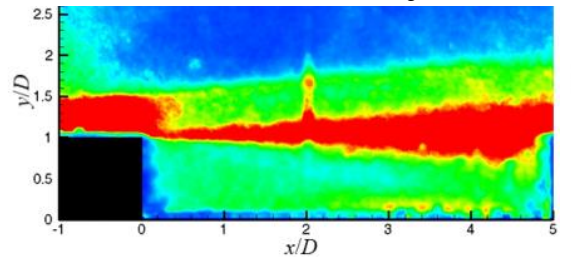

(c) CASE $2(0.5 L, J=1.2)$

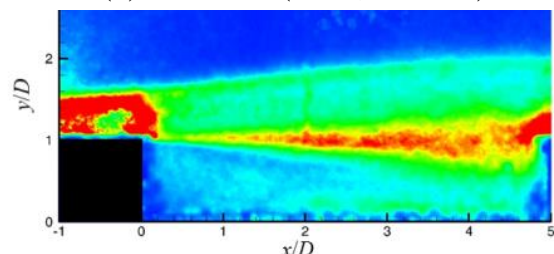

(f) CASE $5(0.5 L, J=2.7)$

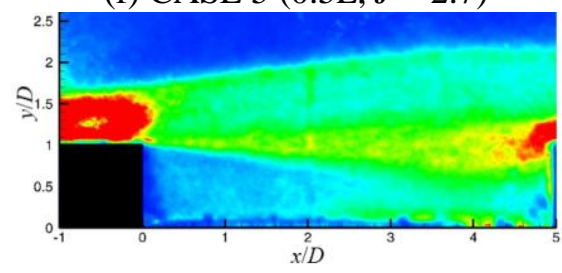

(i) CASE $8(0.5 L, J=5.3)$

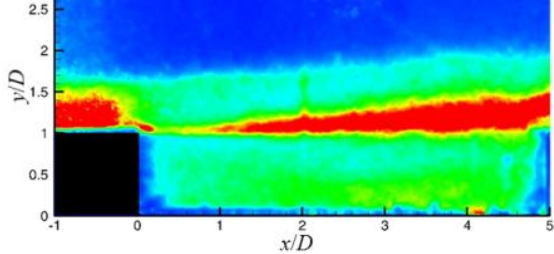

(d) CASE $3(1 L, J=1.2)$

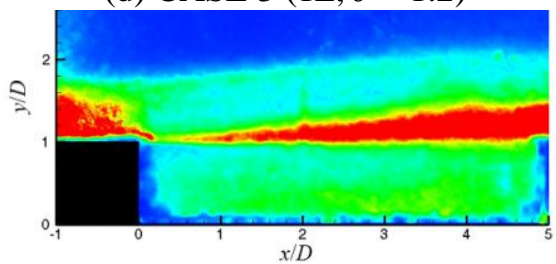

(g) CASE $6(1 L, J=2.7)$

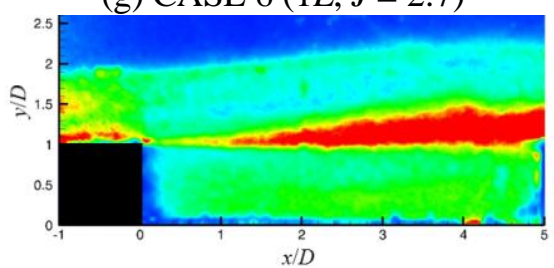

(k) CASE $9(1 L, J=5.3)$

Figure 9: Flow field velocity of root-mean-square contours for the x-direction. 


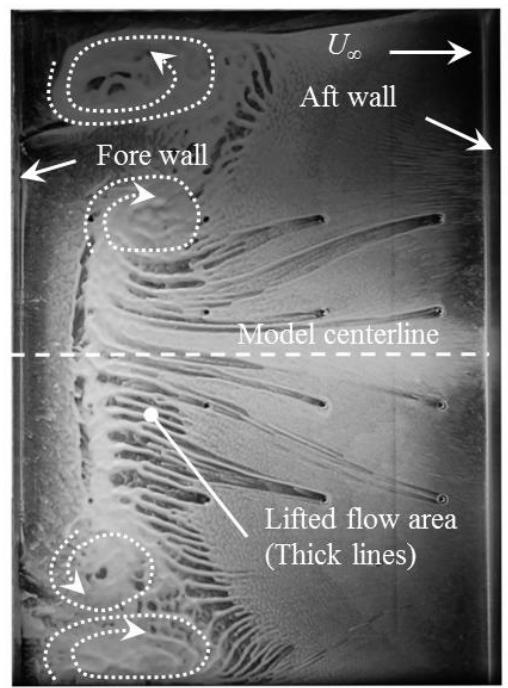

(a) CASE 0 (No jet)

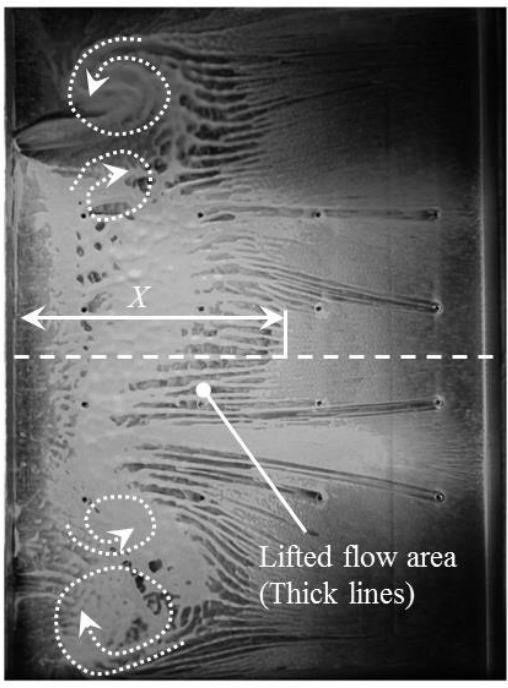

(c) CASE $8(0.5 L, J=5.3)$

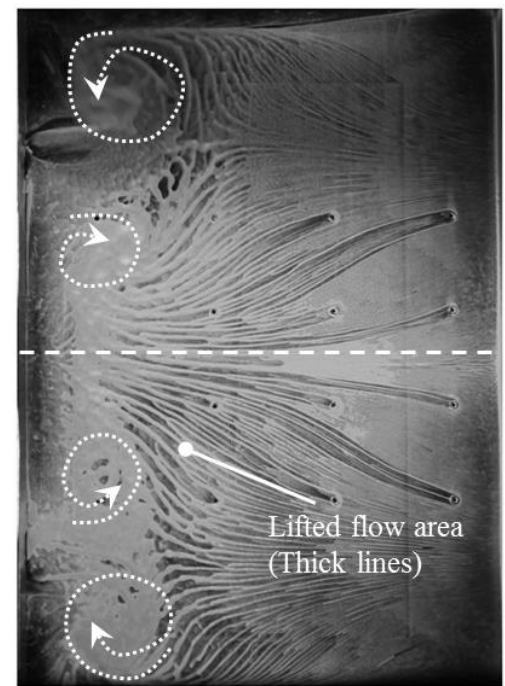

(b) CASE $7(0.1 L, J=5.3)$

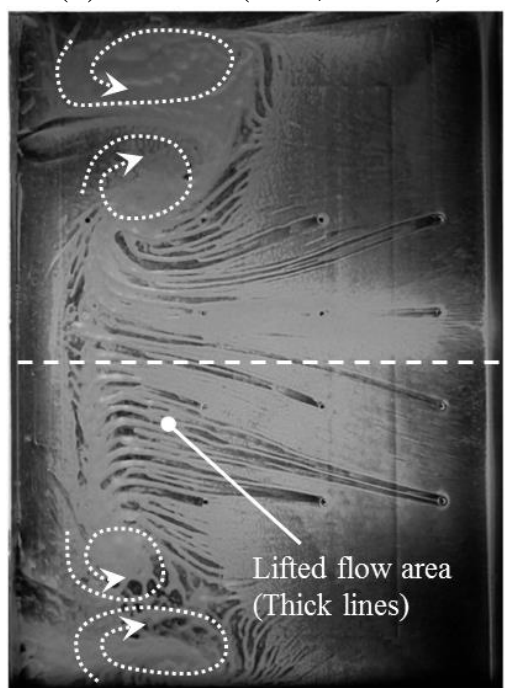

(d) CASE $9(1 L, J=5.3)$

Figure 10: Surface oil flow visualization on the cavity floor.

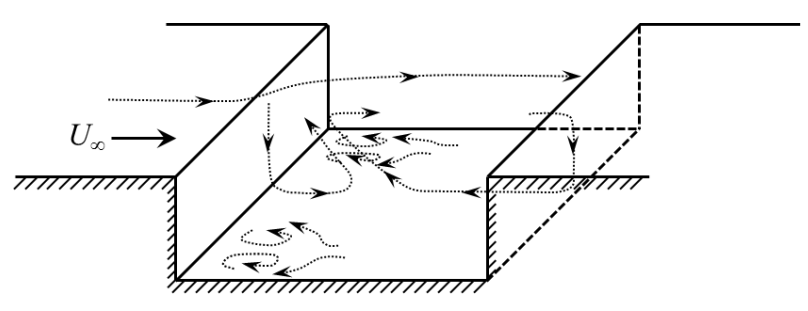

(a) CASE 0 (No jet)

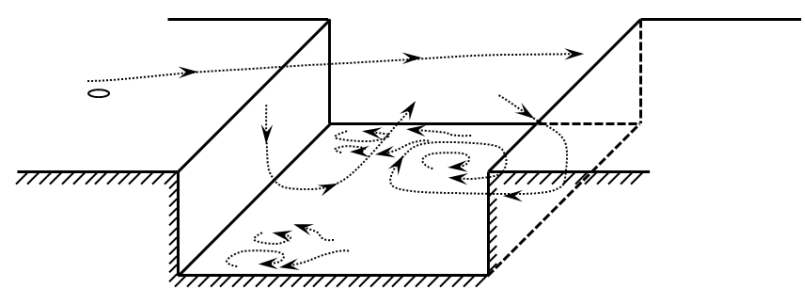

(c) CASE $8(0.5 L, J=5.3)$

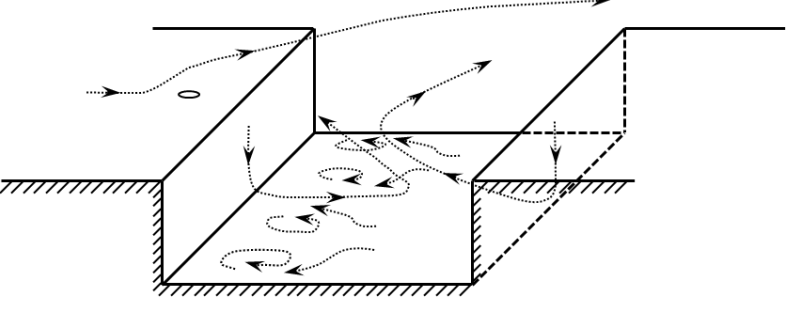

(b) CASE $7(0.1 L, J=5.3)$

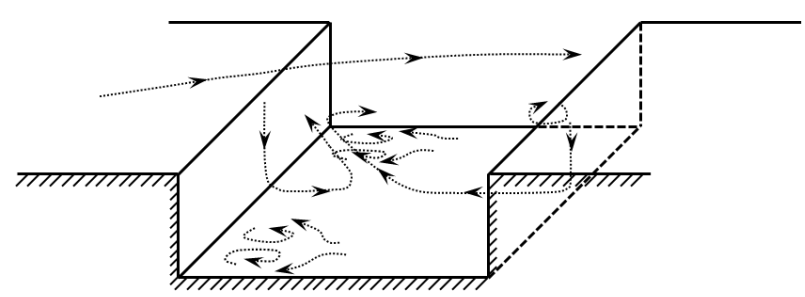

(d) CASE $9(1 L, J=5.3)$

Figure 11: Flow structure schematics around the cavity. 


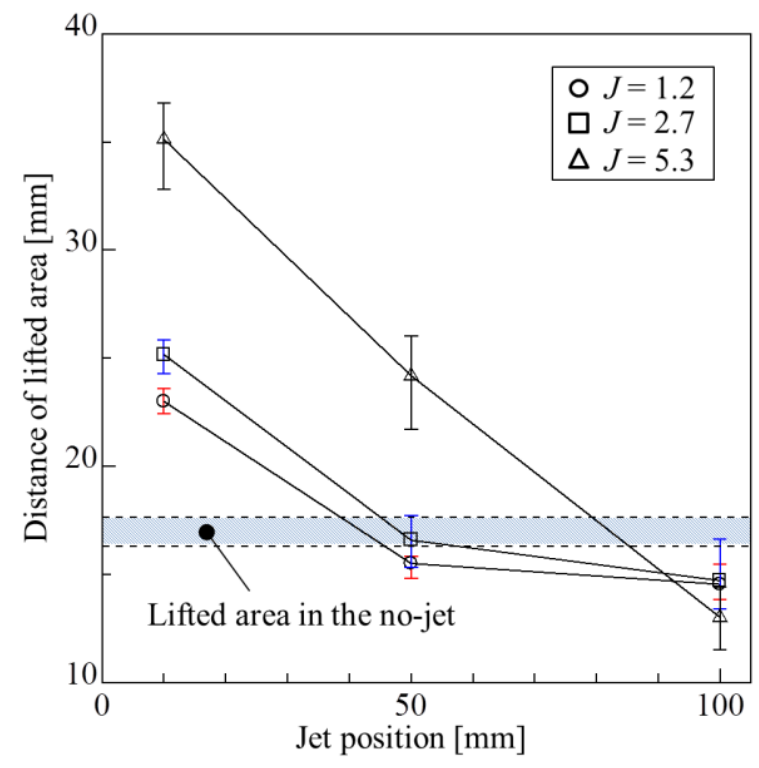

Figure 12: The distance of the flow lifted area.

Tables

Table 1: Test model

\begin{tabular}{|c|c|c|}
\hline Test case & Jet position & $J$ \\
\hline $\begin{array}{c}\text { CASE } 0 \\
\text { (Baseline) }\end{array}$ & $\begin{array}{c}- \\
\text { (No jet) }\end{array}$ & - \\
\hline CASE 1 & $0.1 L(10 \mathrm{~mm})$ & 1.2 \\
\hline CASE 2 & $0.5 L(50 \mathrm{~mm})$ & 1.2 \\
\hline CASE 3 & $1 L(100 \mathrm{~mm})$ & 1.2 \\
\hline CASE 4 & $0.1 L(10 \mathrm{~mm})$ & 2.7 \\
\hline CASE 5 & $0.5 L(50 \mathrm{~mm})$ & 2.7 \\
\hline CASE 6 & $1 L(100 \mathrm{~mm})$ & 2.7 \\
\hline CASE 7 & $0.1 L(10 \mathrm{~mm})$ & 5.3 \\
\hline CASE 8 & $0.5 L(50 \mathrm{~mm})$ & 5.3 \\
\hline CASE 9 & $1 L(100 \mathrm{~mm})$ & 5.3 \\
\hline
\end{tabular}

Table 2: The capability of the seeding particle for the flow-tracing.

\begin{tabular}{|c|c|c|c|c|c|c|}
\hline$P_{0}[\mathrm{kPa}]$ & $T_{0}[\mathrm{~K}]$ & $\delta[\mathrm{mm}]$ & $K n_{d}$ & $\tau_{p}[\mu \mathrm{s}]$ & $\tau_{f}[\mu \mathrm{s}]$ & $S k$ \\
\hline 75 & $13.5 \pm 1.0$ & $6.3 \pm 0.3$ & 0.23 & 4.60 & 122 & 0.04 \\
\hline
\end{tabular}

